\title{
Analysis of Intensity Modulation and Switched Fault Techniques for Different Optical Fiber Cables
}

\author{
Rahul Malhotra (Corresponding author) \\ Department of Electronics and Communication Engineering \\ BMSCE, MUKTSAR, Punjab Technical University \\ Jalandhar, India \\ E-mail: blessurahul@gmail.com \\ Subhash Singh \\ Department of Electronics and Communication Engineering \\ BMSCE, MUKTSAR, Punjab Technical University \\ Jalandhar, India \\ E-mail: subhash_ghoga83@yahoo.co.in \\ Harkirtan Singh \\ Department of Electronics and Communication Engineering \\ BMSCE, MUKTSAR, Punjab Technical University \\ Jalandhar, India \\ E-mail: harkirtan87@gmail.com \\ Parminder Kumar Luthra \\ Department of Electronics and Communication Engineering \\ BMSCE, MUKTSAR, Punjab Technical University \\ Jalandhar, India \\ E-mail: pkluthra@gmail.com
}

Received: July 12, 2011

Accepted: July 20, 2011

doi:10.5539/cis.v4n5p36

\begin{abstract}
Fiber optics is the fastest and reliable means of transferring large amounts of data Optical fiber links are used for local area networks to world wide data communication. Fiber-optic communication is a method of transmitting information from one place to another by sending pulses of light through an optical fiber. Digital optical communications explores the practical applications of this union and applies digital modulation techniques to optical communications systems. Intensity modulation is a modulation technique in which the optical output power of a source is varied in accordance with some characteristic of the modulating signal. Fiber optics trainer ST 2502, which is a single board fiber optic transmitter receiver module providing two independent fiber optic communication links, is used in the present work. It is used to study and analyze the analog \& digital signals modulation in relation to the losses in optical fiber. This work intends to obtain an intensity modulation of a digital signal transmitted over fiber optic cable and demodulate the same at receiver end to get the original signal. Different fiber optic cables namely SIPMMA fiber, thermocouple type $\mathrm{K}$ with glass fiber/stainless cables, OFNR cable is used to obtain intensity modulation using digital input signal. It has been investigated that the output of detector is affected with the change in cable and the size of cables also plays an important role in data transmission. The study of switched faults in intensity modulation is also taken up in the study.
\end{abstract}

Keywords: Fiber optic communication, Intensity modulation, Digital communication 


\section{Introduction}

Communication plays an important role in the transmission of information using the communication devices like mobiles, telephone sets and other mediums like Internet. Communication engineering deals with the transmission of various signals from one point to another. Fiber optics is the fastest and reliable means of transferring large amounts of data. Optical fiber links are used for local area networks to world wide data communication. Optical communication system use high carrier frequencies in the visible or near infrared region of the electromagnetic spectrum.

\section{The Evolution of Fiber Optic System}

A fiber transmission system is often characterized by its bandwidth-distance product, often expressed in units of $\mathrm{MHz} \times \mathrm{km}$. This value is a product of bandwidth and distance because there is a trade off between the bandwidth of the signal and the distance over which the signal can be carried. For example, a common multimode fiber with bandwidth-distance product of $500 \mathrm{MHz} \times \mathrm{km}$ could carry a $500 \mathrm{MHz}$ signal for $1 \mathrm{~km}$ or a $1000 \mathrm{MHz}$ signal for $0.5 \mathrm{~km}$.

Optical fiber was successfully developed in 1970 by Corning Glass Works, with attenuation low enough for communication purposes (about $20 \mathrm{~dB} / \mathrm{km}$ ). GaAs semiconductor lasers that were compact were suitable for transmitting light through fiber optic cables for long distances.

This first-generation system operated at a bit rate of $45 \mathrm{Mbps}$ with repeater spacing of up to $10 \mathrm{~km}$. The second generation of fiber-optic communication developed InGaAsP semiconductor lasers for commercial use in the early $1980 \mathrm{~s}$, operated at $1.3 \mu \mathrm{m}$. These early systems were initially limited by multi mode fiber dispersion, and in 1981 the single-mode fiber was revealed to greatly improve system performance; however practical connectors capable of working with single mode fiber proved difficult to develop. By 1987, these systems were operating at bit rates of up to $1.7 \mathrm{~Gb} / \mathrm{s}$ with repeater spacing up to $50 \mathrm{~km}$. Third-generation fiber-optic systems operated at $1.55 \mu \mathrm{m}$ and had losses of about $0.2 \mathrm{~dB} / \mathrm{km}$ due to pulse-spreading at that wavelength. These losses were overcome by using dispersion-shifted fibers, designed to have minimal dispersion at $1.55 \mu \mathrm{m}$ or by limiting the laser spectrum to a single longitudinal mode. These developments eventually allowed third-generation systems to operate commercially at $2.5 \mathrm{Gbit} / \mathrm{s}$ with repeater spacing in excess of $100 \mathrm{~km}$. The fourth generation of fiber-optic communication systems used optical amplification to reduce the need for repeaters and wavelength-division multiplexing to increase data capacity. These two improvements caused a revolution that resulted in the doubling of system capacity every 6 months starting in 1992 until a bit rate of $10 \mathrm{~Tb} / \mathrm{s}$ was reached by 2001. Recently, bit-rates of up to $14 \mathrm{Tbit} / \mathrm{s}$ have been reached over a single $160 \mathrm{~km}$ line using optical amplifiers.

The focus of development for the fifth generation of fiber-optic communications is on extending the wavelength range over which a WDM system can operate. The conventional wavelength window, known as the $\mathrm{C}$ band, covers the wavelength range 1.53-1.57 $\mu \mathrm{m}$, and the new dry fiber has a low-loss window promising an extension of that range to 1.30-1.65 $\mu \mathrm{m}$. Other developments include the concept of "optical solitons" pulses that preserve their shape by counteracting the effects of dispersion with the nonlinear effects of the fiber by using pulses of a specific shape.

\section{Optical Fiber}

The transmission medium in fiber optic communication system is an optical fiber. The optical fiber is the transparent flexible filament that guides light from a transmitter to a receiver. An optical information signal entered at the transmitter end of a fiber optic communication system is delivered to the receiver end by the optical fiber. The optical fiber provides the connection between a transmitter and a receiver.

The optical fiber is generally made from a type of glass called silica or, less commonly nowadays, from plastics. An optical fiber consists of a core, cladding, and a buffer (a protective outer coating), in which the cladding guides the light along the core by using the method of total internal reflection. The core and the cladding (which has a lower-refractive-index) are usually made of high-quality silica glass, although they can both be made of plastic as well.

\section{Optical Fiber Cables}

There are different types of fiber optic cables available, few of them are discussed here.

\subsection{SI PMMA Cable}

The $1 \mathrm{~mm}$ PMMA step-index plastic optical fibre (SI-POF) is a robust, low-cost, and easy-to-install transmission medium. These unique features make the SI-POF a highly attractive candidate for in-building networks requiring 
data rates in the range of $1 \mathrm{Gbit} / \mathrm{s}$ to about $3 \mathrm{Gbit} / \mathrm{s}$. Due to its large diameter, modal dispersion limits the bandwidth-length product of the SI-POF to approximately $100 \mathrm{MHz} \times 100 \mathrm{~m}$, a relatively poor value compared to copper cable as well as multimode glass fiber.By using spectrally efficient adaptive multiple sub-carrier modulation, even the SI-POF is suitable for $1 \mathrm{Gbit} / \mathrm{s}$ transmission over a distance of $100 \mathrm{~m}$. Similar modulation techniques are already applied in large scale to DSL, WLAN, and WiMax proving its potential for low-cost implementation in high-speed SI-POF networks.

\subsection{GI Cable}

High-bandwidth (2 GHz/km) graded-index polymer optical fiber (GI POF) was successfully obtained by the new interfacial-gel polymerization technique in which the uncreative component was used in order to obtain the quadratic refractive-index distribution. This high-bandwidth GI POF makes it possible to transmit high-speed optical signals in the short range network which was not covered by the step-index type POF commercially available. The high bandwidth GI POF will open the way for great advantages in the high-speed multimedia network.

\subsection{OFNR}

Optical fiber, nonconductive, riser (OFNR) is a type of optical fiber cable. This name is used for interior fiber-optic cables which contain no electrically conductive components, and which are for use in riser applications; they are engineered to prevent the spread of fire from floor to floor in a building. They are distinct from optical fiber nonconductive, plenum cable (OFNP), and general-purpose optical cable. OFNR cables can not be installed in plenum areas since they do not have the required fire and smoking rating as Plenum rated cables. OFNP plenum cables can be used as substitutes for OFNR cables.

\section{Optical Fiber Transmission Link}

The optical Fiber communication is in general possible in any area that requires transfer of information from one place to another place. Optical communication system can be classified into two broad categories:

\subsection{Unguided Light Wave System}

In unguided optical communication system, the optical beam emitted by the transmitter spread in space. However, unguided optical system is less suitable for broadcasting application because optical beam spreads mainly in forward direction. Their use generally requires accurate pointing between the transmitter and receiver.

\subsection{Guided Light Wave System}

In guided light wave system, the optical beam emitted by the transmitter remains spatially confined. All guided optical system currently used optical fiber.

\section{Digital optical communication}

Digital optical communications explores the practical applications of this union and applies digital modulation techniques to optical communications systems. A modern alternative to sending (binary) digital information via electric voltage signals is to use optical signals. Electrical signals from digital circuits may be converted into discrete optical signals with LEDs or solid-state lasers. Likewise, light signals can be translated back into electrical form through the use of photodiodes or phototransistors for introduction into the inputs of gate circuits. (See Figure 1)

Transmitting digital information in optical form may be done in open air, simply by aiming a laser at a photo detector at a remote distance, but interference with the beam in the form of temperature inversion layers, dust, rain, fog, and other obstructions can present significant engineering problems. (See Figure 2)

A modern alternative to sending (binary) digital information via electric voltage signals is to use optical (light) signals. Electrical signals from digital circuits (high/low voltages) may be converted into discrete optical signals (light or no light) with LEDs or solid-state lasers. Likewise, light signals can be translated back into electrical form through the use of photodiodes or phototransistors for introduction into the inputs of gate circuits.

\section{Modulation}

In order to make light carry a signal, you have to introduce systematic variations (Modulation) in the light to represent the signal. Then, when the light is received you must decode it in such a way as to reconstruct the original signal.

\subsection{Amplitude Modulation (AM)}

If the signal in Figure 3 is amplitude modulated by a sine wave as shown in Figure 4, sidebands are produced in 
the frequency domain at $\mathrm{Fc} \pm \mathrm{FAM}$. AM other than by a pure sine wave will cause additional sidebands normally at $\mathrm{Fc} \pm \mathrm{nFAM}$, where $\mathrm{n}$ equals $1,2,3$, 4, etc.

The need for advanced transmission techniques over long haul optically amplified communications has prompted a convergence of digital and optical communications. Digital optical communications explores the practical applications of this union and applies digital modulation techniques to optical communications systems. A modern alternative to sending (binary) digital information via electric voltage signals is to use optical (light) signals. Electrical signals from digital circuits (high/low voltages) may be converted into discrete optical signals (light or no light) with LEDs or solid-state lasers.

\subsection{Frequency Modulation (FM)}

As shown in Figure 4, an unmodulated RF signal in the time domain has only a single spectral line at the carrier frequency (fc) in the frequency domain. If the signal is frequency modulated, as shown in Figure 5, the spectral line will correspondingly shift in the frequency domain.

\section{Intensity Modulation}

In optical communications, a form of modulation in which the optical power output of a source is varied in accordance with some characteristic of the modulating signal. In intensity modulation, there are no discrete upper and lower sidebands in the usually understood sense of these terms, because present optical sources lack sufficient coherence to produce them. The envelope of the modulated optical signal is an analog of the modulating signal in the sense that the instantaneous power of the envelope is an analog of the characteristic of interest in the modulating signal. Recovery of the modulating signal is by direct detection, not heterodyning.

An intensity modulation optical communication apparatus including a transmitter unit for producing a single side-band intensity modulated light signal based on a carrier light and a transmission signal, and a receiver unit for receiving the single side-band intensity modulated light signal and reproducing the transmission signal there from, wherein said transmitter unit comprises carrier light producing means for producing a carrier light according to a control signal; control signal generator means for producing said control signal by phase-shifting said transmission signal by a constant phase angle and emphasizing a high frequency component thereof; and amplitude modulation means for amplitude-modulating said carrier light according to said transmission signal to output said single side-band intensity modulated light signal, and wherein the receiver unit comprises: means for producing an electric signal by detecting the single side-band intensity modulated light signal; and equalizer means for equalizing the electric signal to reproduce the transmission signal

\section{Digital Modulation}

With digital modulation, discrete changes in light intensity are obtained (i.e. ON-OFF Pulses) Figure 6 shows a block schematic of a typical digital optical fiber link. Initially, input digital signal from the information source is suitably encoded for optical transmission. The LED drive circuit directly modulates the intensity of the light with encoded digital signal. Hence, a digital optical signal is launched into the optical fiber cable. An amplifier to provide gain follows the phototransistor used as detector. Finally the signal obtained is decoded to give the original digital information. Digital Bias Voltage: Incase of a digital signal the only information which needs to be conveyed is the ON state and OFF state.

\section{Fiber optic Trainer ST 2502}

It is a single board fiber optic transmitter - receiver module providing two independent fiber optic communication links. It may be used as a stand alone system, allowing studying and illustrating Amplitude Modulation using analog \& digital signals frequency \& pulse width modulation, losses in optical fiber. The module may be configured to establish an analog link also it may be used to provide a full two serial fiber optic communication link between two micro computers. ST2502 board accepts the information signal and converts it in to a suitable form for transmission through the fiber optic links.

\section{Case I}

The data transmission efficiency of different fiber optic cables for Intensity modulated digital optical communication is tested using the Fiber Optic Trainer ST2502. In this paper, the three different types of cables are compared for their performance. The input and output signals are compared as shown in the Table 1 and Table 2.

\section{Case II}

There are various types of faults that occur during the transmission and reception of a signal, the most common faults that can occur are tested using fiber optic trainer ST2502. In this trainer, the eight types of switch faults are 
designed for study, the switched fault 1 and switched fault 5 using three different types of cables are taken up for the present work. The different cables used for the study are $1 \mathrm{~m}$ SI PMMA cable, $2 \mathrm{~m}$ glass SI cable and $1 \mathrm{~m}$ GI cable. The experimental results are as shown in Table 3 and Table 4.

\section{Conclusion}

The data transmission efficiency and switched faults for different fiber optic cables using Intensity modulated digital optical communication is tested using the Fiber Optic Trainer ST2502. The different types of cables are compared for their performance. The input and output signals are compared which shows the better signal transfer using $1 \mathrm{~m}$ GI Cable as compared to $1 \mathrm{~m}$ SI PMMA and $1 \mathrm{~m}$ OFNR cables but on the other hand $2 \mathrm{~m}$ SI PMMA cable shows better results when compared with $2 \mathrm{~m}$ GI and $2 \mathrm{~m}$ OFNR cables. This shows that for small length of cable, GI cable gives better results but SI PMMA cable shows good response for long length of the cable. The switched fault comparison for SW1 type of fault shows the distortion in output signal while the second type of fault viz. SW5 fault affects the tunability of the control parameters.

\section{References}

B. Forzouan. Data Communications and Networking. 3rd Edition. McGraw Hill Publishers.

C. M. Khalique \& A. Biswas. (2009). A Lie symmetry approach to nonlinear Schrödinger's equation with non-Kerr law nonlinearity. Elsevier, Commun Nonlinear Sci Numer Simulate, 14, 4033-4040.

E. Kengne \& R. Vaillancourt. (2009). Transmission of solitary pulse in dissipative nonlinear transmission lines. Elsevier, commun nonlinear sci numer simulate, 14, 3804-3810.

Ezra Ip, Alan Pak Tao Lau, Daniel J. F. Barros \& Joseph M. Kahn. Coherent detection in optical fiber systems, 16(2), 753-791.

F. Zeng \& J. Yao. (2005). All-Optical Microwave Mixing \& Bandpass Filtering in a Radio-Over-Fiber Link. IEEE photonics technology letters, 17, 4.

H. H. Lu, C. Yi Li, S.J. Tzeng, H. C. Peng \& Wen-I Lin, (2009). Full-duplex radio-on-fiber transport systems based on main and multiple side modes injection-locked DFB laser diode. Optical Fiber Technology, 15, 251-257. doi:10.1016/j.yofte.2008.11.003, http://dx.doi.org/10.1016/j.yofte.2008.11.003.

J. G. Liu. (2000). Smoothing Filter-based Intensity Modulation: A spectral preserve image fusion technique for improving spatial details, 21, 3461-3472.

J. He, L. Chen, Z. Dong, S. Wen \& J. Yu (2009). Full-duplex radio-over-fiber system with photonics frequency quadruples for optical millimeter-wave generation. Optical Fiber Technology, 15, 290-295. doi:10.1016/j.yofte.2008.12.006, http://dx.doi.org/10.1016/j.yofte.2008.12.006.

K. Blary, S. Dupont, J Vilcot, E Mollot, D. Decoster \& J. Chazelas. (2002). DOS optical switch for microwave optical links based applications. IEEE, electronics letters, 38, 25.

K. Suzuki, H. Kubota, S. Kawanishi, M.i Tanaka \& M. Fujita. (2001). Optical properties of a low-loss polarization-maintaining photonic crystal fiber. Optics Express, 9(13), 676-680. doi:10.1364/OE.9.000676, http://dx.doi.org/10.1364/OE.9.000676.

L. D. Garrett, M. H. Eiselt, J. M. Wiesenfeld, M. R. Young \& R. W. Tkach. (2003). Bidirectional ULH Transmission of $160-\mathrm{Gb} / \mathrm{s}$ Full-Duplex Capacity Over 5000km in a Fully Bidirectional Recirculating Loop. IEEE Journal of Lightwave Technology, 16(7), 1757-1759.

M. Ahmed \& A. E. LAFI. (2008). Analysis of small-signal intensity modulation of semiconductor asers taking account of gain suppression. Indian Academy of Sciences, 71, 99-115.

M. Daikoku, T. Miyazaki, I. Morita, H. Tanaka, F. Kubota \& M. Suzuki. (2006). 8 X 160-Gb/s WDM Field Transmission Experiment with Single-Polarization RZ-DPSK Signals and PMD Compensator. IEEE Photonics Technology Letters, 18(2), 391-393, 15.

M. Kumar, A. K. Sharma \& T.S. Kamal. (2007). Significance of prechirping on long-haul pathaveraged soliton impulse in re-circulating loop at 10 and $20 \mathrm{~Gb} / \mathrm{s}$ with TOD. Opt. Int. J. Light Electron, 10, 665-667.

M. Nakazawa, E. Yamada, H. Kubota \& K. Suzuki. (1991). 10 Gbit/s Soliton data transmission over one million kilometers. IEEE, electronics letters, 27, 14.

M. Nakazawa \& H. Kubota. (1995), Optical soliton communication in a positively and negatively dispersion allocated optical fiber transmission line. IEEE, electronics letters, 31, 3. 
Okoshi, T. (1985). Polarization-state control schemes for heterodyne or homodyne optical fiber communications. $3(6), 1232-1237$.

P. Goldgeier \& G. Eisenstein. (1999). Broad-Band Microwave-Optical Fiber Links Transmitting over Long Distances with Optical Amplification. IEEE microwave and guided wave letters, 9, 1.

Reginald T. Cahill. (2007). Optical-Fiber Gravitational Wave Detector: Dynamical 3-Space Turbulence Detected. Progress In Physics, 4.

T. A. Birks, J. C. Knight \& P. St. J. Russell. (1997). Endlessly single-mode photonic crystal fiber. Optics Letters, 22, 961-963. doi:10.1364/OL.22.000961, http://dx.doi.org/10.1364/OL.22.000961.

W. K. Choi, D. G. Kim, D. G. Kim, Y. W. Choi, K. D. Choquette, S. Lee \& D. H. Woo. (2006). Optical AND/OR gates based on monolithically integrated vertical cavity laser with depleted optical thyristor structure. Opt. Express, 14, 11833-11838. doi:10.1364/OE.14.011833, http://dx.doi.org/10.1364/OE.14.011833.

W. Wang, H. N. Poulsen, L. Rau, H. F. Chou, J. E. Bowers \& D. J. Blumenthal. (2005). Raman-Enhanced Regenerative Ultrafast All-Optical Fiber XPM Wavelength Converter. Journa of Lightwave Technology, 23(3).

Yasin M. Karfaa, M. Ismail \& Abbou F. M. (2007). Effects of Four-wave Mixing Crosstalk in WDM Networks on the Transmitted Optical Frequencies and Wavelengths of Channels for Various Fiber Types. Asia pacific conference on applied electromagnetic proceedings.

Table 1. Comparison of 1m SI PMMA, 1m GI and 1m OFNR Cables

\begin{tabular}{|l|l|l|l|}
\hline Cable & Attribute & Input & Output \\
\hline \multirow{3}{*}{$1 \mathrm{~m}$ SI PMMA } & Amp & $5 \mathrm{~V}$ & $0.1 \mathrm{~V}$ \\
\cline { 2 - 4 } & Time & $2 \mathrm{~ms}$ & $2 \mathrm{~ms}$ \\
\hline \multirow{3}{*}{$1 \mathrm{~m}$ GI } & Amp & $5 \mathrm{~V}$ & $5 \mathrm{~V}$ \\
\cline { 2 - 4 } & Time & $2 \mathrm{~ms}$ & $2 \mathrm{~ms}$ \\
\hline \multirow{2}{*}{$1 \mathrm{~m}$ OFNR } & Amp & $5 \mathrm{~V}$ & St.hor.line \\
\cline { 2 - 4 } & Time & $2 \mathrm{~ms}$ & \\
\hline
\end{tabular}

Table 2. Comparison of 2m SI PMMA, 2m GI Cables and 2m OFNR Cables

\begin{tabular}{|l|l|l|l|}
\hline Cable & Attribute & Input & Output \\
\hline \multirow{2}{*}{$2 \mathrm{~m}$ Glass SI } & Amp & $5 \mathrm{~V}$ & $5 \mathrm{~V}$ \\
\cline { 2 - 4 } & Time & $10 \mathrm{~ms}$ & $10 \mathrm{~ms}$ \\
\hline \multirow{2}{*}{$2 \mathrm{~m}$ GI } & Amp & $5 \mathrm{~V}$ & $3.0 \mathrm{~V}$ \\
\cline { 2 - 4 } & Time & $1.6 \mathrm{~ms}$ & $1.6 \mathrm{~ms}$ \\
\hline \multirow{2}{*}{$2 \mathrm{~m}$ OFNR } & Amp & $5 \mathrm{~V}$ & St.hor.line \\
\cline { 2 - 3 } & Time & $10 \mathrm{~ms}$ & \\
\hline
\end{tabular}


Table 3. Comparison of $1 \mathrm{~m}$ SI PMMA, $1 \mathrm{~m}$ GI and 2m Glass SI Cables for switched fault SW1

\begin{tabular}{|c|c|c|c|c|}
\hline Cable & Attribute & $\begin{array}{l}\text { Input of } \\
\text { Emitter }\end{array}$ & $\begin{array}{l}\text { Output of Amplifier } \\
\text { SW1 OFF }\end{array}$ & $\begin{array}{c}\text { Output of Amplifier } \\
\text { SW1 ON }\end{array}$ \\
\hline \multirow[t]{2}{*}{ 1m SI PMMA } & Amp & $5 \mathrm{~V}$ & $1.2 \mathrm{~V}$ & \multirow{2}{*}{$\begin{array}{l}\text { Distortion in Output } \\
\text { Signal }\end{array}$} \\
\hline & Time & 2 msec. & $2 \mathrm{msec}$. & \\
\hline \multirow[t]{2}{*}{ 2m Glass SI } & Amp & $5 \mathrm{~V}$ & $5 \mathrm{~V}$ & \multirow{2}{*}{$\begin{array}{l}\text { Distortion in Output } \\
\text { Signal }\end{array}$} \\
\hline & Time & $2 \mathrm{msec}$. & $2 \mathrm{msec}$. & \\
\hline \multirow[t]{2}{*}{$1 \mathrm{~m} \mathrm{GI}$} & Amp & $5 \mathrm{~V}$ & $5 \mathrm{~V}$ & \multirow{2}{*}{$\begin{array}{l}\text { Distortion in Output } \\
\text { Signal }\end{array}$} \\
\hline & Time & $2 \mathrm{msec}$ & $2 \mathrm{msec}$ & \\
\hline
\end{tabular}

Table 4. Comparison of $1 \mathrm{~m}$ SI PMMA, $1 \mathrm{~m}$ GI and 2m Glass SI Cables for switched fault SW5 when emitter input is set to $4 \mathrm{~V}$ peak to peak

\begin{tabular}{|c|c|c|c|c|}
\hline Cable & Attribute & Input & $\begin{array}{l}\text { Output of Amplifier } \\
\text { SW5 OFF }\end{array}$ & $\begin{array}{c}\text { Output of Amplifier SW } \\
5 \text { ON }\end{array}$ \\
\hline \multirow[t]{2}{*}{ 1m SI PMMA } & Amp & $2.8 \mathrm{~V}$ & $0.8 \mathrm{~V}$ & \multirow{2}{*}{$\begin{array}{l}0.04 \mathrm{~V} \text { (No effect of } \\
\text { changing amplitude preset) }\end{array}$} \\
\hline & Time & $2 \mathrm{~ms}$ & $2 \mathrm{~ms}$ & \\
\hline \multirow[t]{2}{*}{$2 \mathrm{~m}$ Glass SI } & Amp & $4 \mathrm{~V}$ & $3.0 \mathrm{~V}$ & \multirow{2}{*}{$\begin{array}{l}2.7 \mathrm{~V} \text { (No effect of } \\
\text { changing amplitude preset) }\end{array}$} \\
\hline & Time & $11 \mathrm{~ms}$ & $11 \mathrm{~ms}$ & \\
\hline \multirow[t]{2}{*}{$1 \mathrm{~m} \mathrm{GI}$} & Amp & $4 V$ p-p & $2 \mathrm{~V}$ & \multirow{2}{*}{$\begin{array}{l}1.6 \mathrm{~V} \text { (No effect of } \\
\text { changing amplitude preset) }\end{array}$} \\
\hline & Time & $2 \mathrm{~ms}$ & $2 \mathrm{~ms}$ & \\
\hline
\end{tabular}

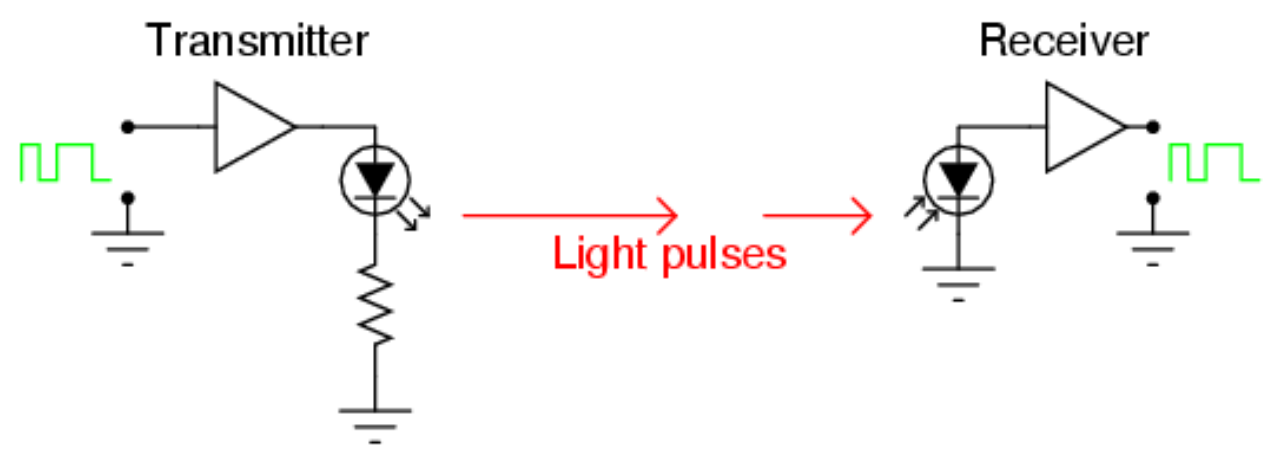

Figure 1. Transmitter and Receiver with Light Pulses 


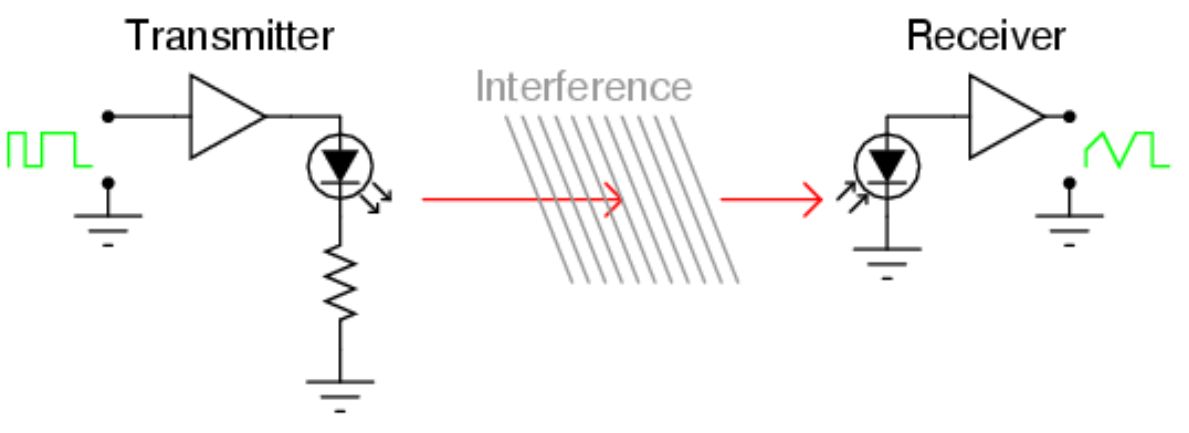

Figure 2. Transmitter and Receiver With Interference

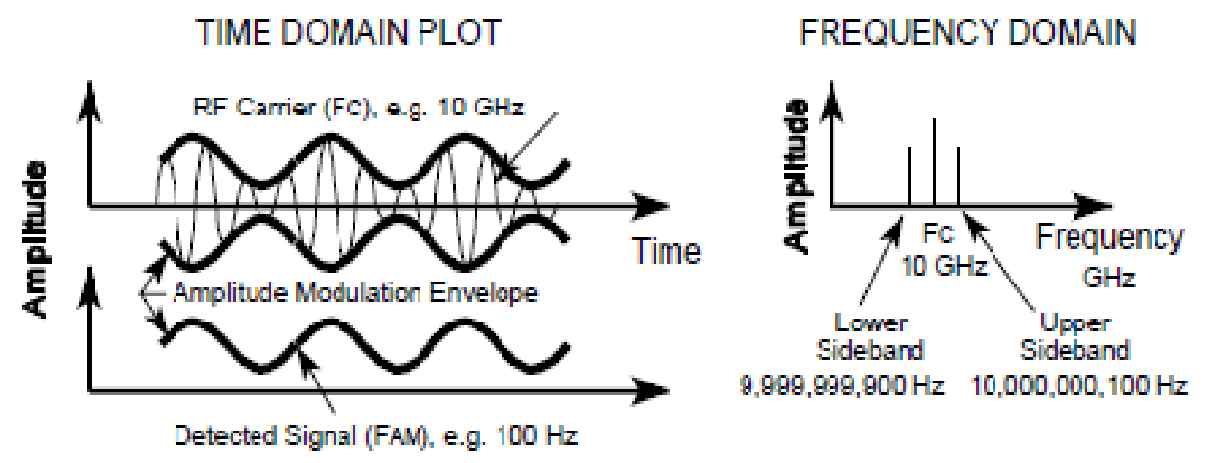

Figure 3. Sinewave Modulated Rf Signal

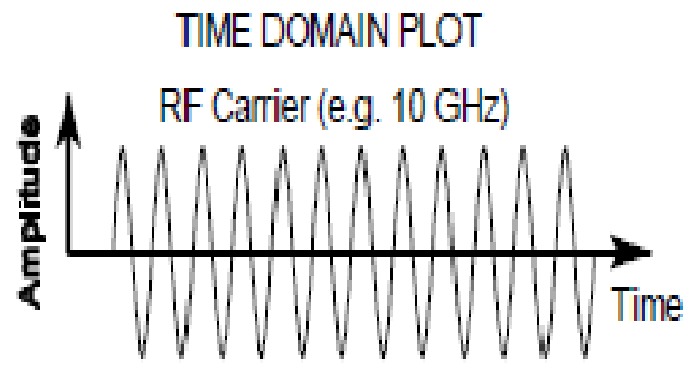

\section{FREQUENCY DOMAIN}

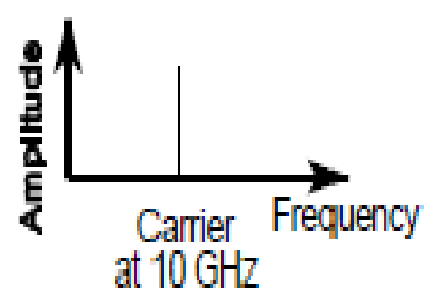

Figure 4. Unmodulated RF Signal 


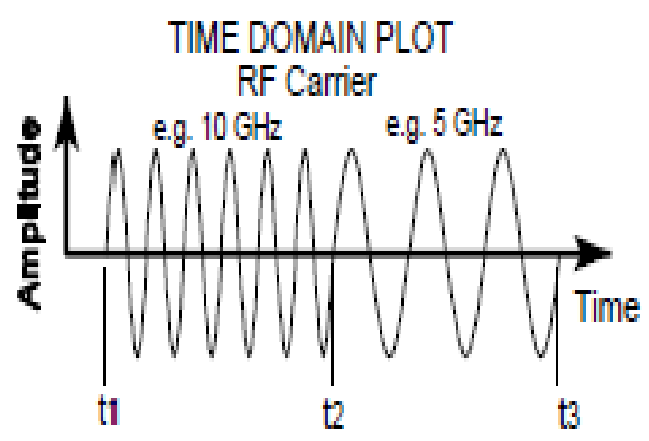

\section{FREQUENCY DOMAIN}

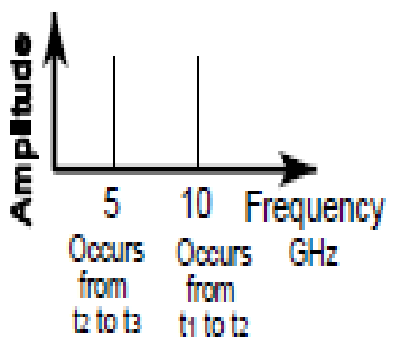

Figure 5. RF Signal with Frequency Modulation

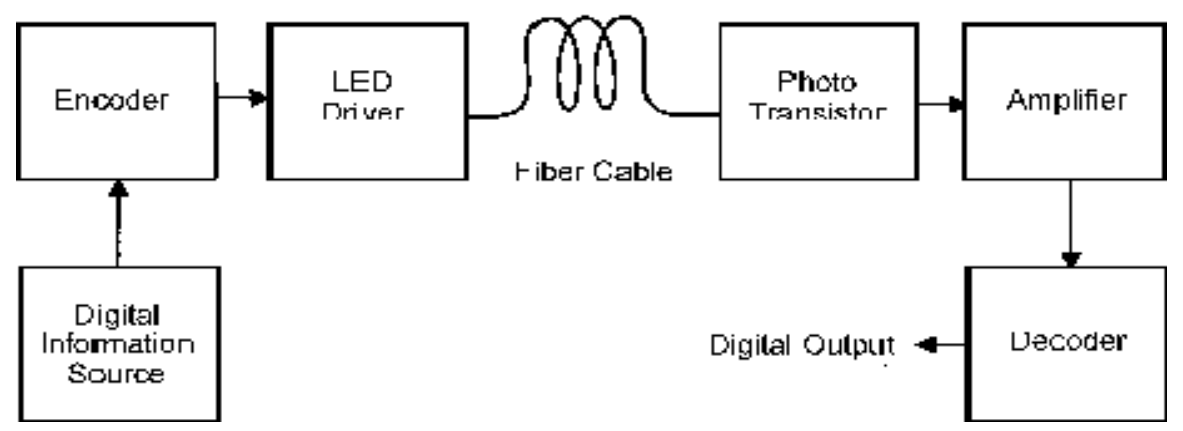

Figure 6. Block Schematic of a Typical Digital Optical Fiber Link 